\title{
A espiritualidade no cuidado em saúde na Atenção Primária
}

\author{
The spirituality in health care in the Primary Health \\ Espiritualidad en la atención de salud en Atención Primaria
}

\section{Luiz Augusto de Azevedo Pinheiro', Sandra Maria Barroso Werneck Vilagra ${ }^{2}$, Cristina Fidalgo Affonso Pinheiro ${ }^{3}$, Eduardo Herrera Rodrigues de Almeida Júnior ${ }^{4}$, Maria Cristina Almeida de Souza $^{5}$}

Como citar esse artigo. Pinheiro, LAA; Vilagra, SMBW; Pinheiro, CFA; Almeida Junior, EHR; de Souza, MCA. A espiritualidade no cuidado em saúde na Atenção Primária. Revista PróUniverSUS. 2019 Jul./Dez.; 10 (2): 7074 .

\section{Resumo}

Introdução: Atenção especial tem sido dispensada à espiritualidade como estratégia de enfrentamento de doenças, pois tem papel protetor contra a morbidade psicológica, tendo em vista seu impacto sobre a maneira como a pessoa lida com a patologia. Metodologia: aborda-se, por meio da revisão de literatura, a espiritualidade na avaliação clínica dos pacientes pelos profissionais de saúde, cujo conhecimento da dimensão espiritual do paciente é capaz de contribuir para o alívio do sofrimento e enfrentamento da doença. Resultados: foram localizadas 11 publicações que atenderam aos critérios de inclusão, sendo 7 artigos, 3 livros, 1 dissertação. Discussão: a Estratégia Saúde da Família (ESF), democratizou o acesso aos serviços de saúde no nível primário de Atenção à Saúde. Atuam nas Unidades de Atenção Primária à Saúde, profissionais generalistas, aos quais compete prestarem um cuidado capaz de resolver aproximadamente $80 \%$ das demandas, cujo componente espiritual deve ser considerado. Imprescindível inclusão da espiritualidade na avaliação clínica dos usuários das Unidades de APS, tendo por pressuposto que o preconizado vínculo médico-paciente poderá otimizar a escuta qualificada, a compreensão pelo profissional do significado do adoecer para o paciente, bem como o estabelecimento de um plano terapêutico capaz de considerar especificidades espirituais e religiosas do paciente, favorecendo o prognóstico e o enfrentamento das adversidades, decorrentes do adoecimento. Considerações finais: considerando que a dimensão espiritual tem uma natureza sistêmica, é fundamental sua utilização pelos profissionais da APS. Há necessidade de mudança de paradigma no atendimento a saúde, com afastamento da visão biomédica cartesiana tecnicista para visão de espiritualidade e saúde.

Palavras-chave: Espiritualidade, Religião, Medicina, Relação médico-paciente.

\begin{abstract}
Introduction: attention has been given to spirituality as a strategy for coping with illnesses, as it has a protective role against psychological morbidity, given its impact on the way people deal with pathology. Methodology: through the literature review, we approach spirituality in the clinical evaluation of patients by health professionals, whose knowledge of the patient's spiritual dimension is able to contribute to the relief of suffering and coping with the disease. Results: 11 publications were found that met the inclusion criteria, 7 articles, 3 books, 1 dissertation. Discussion: the Family Health Strategy, democratized access to health services at the primary level of Health Care. Primary Health Care Units, generalist professionals, who are responsible for providing care capable of solving approximately $80 \%$ of the demands, whose spiritual component must be considered. It is essential to include spirituality in the clinical evaluation of users of Primary Health Care Units, assuming that the recommended doctor-patient bond can optimize qualified listening, the professional's understanding of the meaning of falling ill for the patient, as well as the establishment of a therapeutic plan. Able to consider the patient's spiritual and religious specificities, favoring the prognosis and coping with the adversities resulting from the illness. Final considerations: considering that the spiritual dimension has a systemic nature, its use by Primary Health Care professionals is fundamental. There is a need for a paradigm shift in health care, moving away from the technical Cartesian biomedical view to the view of spirituality and health.
\end{abstract}

Keywords: Spirituality, Religion, Medicine, Physician-Patient Relations.

Afiliação dos autores:1. Médico Residente em Medicina de Família e Comunidade. Hospital Universitário de Vassouras. Vassouras, RJ, Brasil. Email: crislupin@hotmail.com ORCID: https://orcid.org/0000-0003-4185-0944

2. Coordenadora da Residência Médica do Hospital Universitário de Vassouras. Vassouras, RJ, Brasil. Email: sm.vilagra@uol.com.br ORCID: https://orcid.org/0000-0002-61997954

3. Preceptora da Residência em Medicina de Família e Comunidade do Hospital Universitário de Vassouras. Vassouras, RJ, Brasil. Email: cristinadevassouras@gmail.com ORCID https://orcid.org/0000-0002-6686-3381

4. Docente do Curso de Medicina da Universidade de Vassouras. Supervisor da Residência em Medicina de Família e Comunidade do Hospital Universitário de Vassouras. Vassouras, RJ, Brasil. Email: herreratjf@hotmail.com ORCID: https://orcid.org/0000-0001-9628-5757

5. Docente do Curso de Medicina e do Mestrado em Ciências Aplicadas em Saúde da Universidade de Vassouras, RJ, Brasil. Email: mcas.souza@uol.com.br ORCID: https://orcid. org/0000-0001-7631-723X 


\section{Resumen}

Introducción: se ha prestado atención a la espiritualidad como estrategia para hacer frente a las enfermedades, ya que tiene un papel protector contra la morbilidad psicológica, dado su impacto en la forma en que las personas lidian con la patología. Metodología: a través de la revisión de la literatura, abordamos la espiritualidad en la evaluación clínica de los pacientes por parte de profesionales de la salud, cuyo conocimiento de la dimensión espiritual del paciente puede contribuir al alivio del sufrimiento y hacer frente a la enfermedad. Resultados: se encontraron 11 publicaciones que cumplían con los criterios de inclusión, 7 artículos, 3 libros, disertación 1. Discusión: la Estrategia de Salud Familiar (FHS), acceso democratizado a los servicios de salud en el nivel primario de Atención de Salud. Unidades de Atención Primaria de Salud, profesionales generalistas, responsables de brindar atención capaz de resolver aproximadamente el $80 \%$ de las demandas, cuyo componente espiritual debe ser considerado. Es esencial incluir la espiritualidad en la evaluación clínica de los usuarios de las Unidades de APS, suponiendo que el vínculo médico-paciente recomendado pueda optimizar la escucha calificada, la comprensión del profesional del significado de enfermarse para el paciente, así como el establecimiento de un plan terapéutico. Capaz de considerar las especificidades espirituales y religiosas del paciente, favoreciendo el pronóstico y afrontando las adversidades resultantes de la enfermedad. Consideraciones finales: Considerando que la dimensión espiritual tiene una naturaleza sistémica, su uso por parte de los profesionales de la APS es fundamental. Existe la necesidad de un cambio de paradigma en el cuidado de la salud, alejándose de la visión biomédica cartesiana técnica hacia la visión de la espiritualidad y la salud.

Palabras clave: Espiritualidad, Religión, Medicina, Relaciones Médico-paciente.

\section{Introdução}

Estudos epidemiológicos realizados nas últimas décadas apontam para a relação positiva entre espiritualidade, religiosidade e melhores indicadores de saúde. A espiritualidade, força capaz de auxiliar o indivíduo, família e a comunidade a superar as dificuldades da vida e as doenças que vivenciam, é capaz de contribuir para um melhor enfrentamento da realidade cotidiana. ${ }^{1}$ É entendida como uma busca pessoal para entender questões relacionadas ao fim da vida, bem como ao seu sentido e também sobre as relações com o aquilo que, sagrado ou transcendente, pode levar ao desenvolvimento de práticas religiosas ou a formação de comunidades religiosas. ${ }^{2}$

Por religião entende-se um sistema organizado de crenças, rituais e símbolos designados para facilitar o acesso ao que é sagrado/transcendente enquanto religiosidade é definida como a intensidade com que o indivíduo acredita, segue e pratica na religião. Pode ser organizacional - como a participação na igreja ou templo religioso - ou não organizacional, representada pelo ato de rezar, ler livros, assistir programas religiosos na televisão, por exemplo. ${ }^{2}$

Já o Coping Religiosidade/Espiritualidade(CRE) é definido como uso de crenças e comportamentos religiosos capazes de facilitar a solução de problemas, de aliviar ou prevenir as consequências emocionais de circunstâncias de vida estressantes. O estresse, de origem multifatorial, está presente no cotidiano da vida do ser humano. A exposição frequente, intensa ou crônica ao estresse está associada a numerosos efeitos adversos na saúde fica e mental. O que faz diferença no funcionamento humano é a maneira como as pessoas manejam esse estresse, processo conceituado como Coping. Uma das maneiras de manejar o estresse acontece por meio da religião. O CRE relaciona-se, portanto,ao modo como os indivíduos utilizam sua fé para lidar com os fatores estressores eos problemas de suas vidas, tanto emocionais como físicos. Os objetivos do CRE coadunam-se aos objetivos chave da religião que são a busca por significado, controle, conforto espiritual, intimidade com Deus e com outros membros da sociedade, transformação de vida e bem estar físico, psicológico e emocional. ${ }^{3}$

$\mathrm{Na}$ última década, atenção maior tem sido dispensada à espiritualidade e ao seu papel como estratégia de enfrentamento utilizada por pessoas acometidas por doenças.Afinal, a espiritualidade - e também a religião - mostra-se importante diante de enfermidades ao desempenhar papel protetor contra a morbidade psicológica, pois tem impacto importante sobre a maneira como a pessoa lida com a patologia., ${ }^{4,5}$

Cada indivíduo expressa a espiritualidade à sua maneira, relacionando-a à esperança de sobreviver a doença, que amedronta, enquanto a espiritualidade renova, demonstrando a importância do reconhecimento da mesma como estratégia de combate às adversidades e também no planejamento da assistência ao indivíduo doente. ${ }^{6}$

Assim, salienta-se a relevância da espiritualidade na avaliação clínica dos pacientes pelos profissionais de saúde, cujo conhecimento da dimensão espiritual do paciente, representa um diferencial capaz de contribuir para o alívio do sofrimento.

A Constituição Brasileira ${ }^{7}$ determina que saúde é um direito de todos e um dever do Estado, tendo a Estratégia Saúde da Família (ESF), modalidade preferencial de Atenção Primária à Saúde (APS), instituída em 1994, democratizado o acesso aos serviços de saúde no nível primário de Atenção à Saúde. Atuam nas Unidades de APS profissionais generalistas, aos quais compete prestaremum cuidado resolutivo, capaz de resolver aproximadamente $80 \%$ das demandas das pessoas, em sua maioria necessidades clínicas básicas, 
cujo componente espiritual deve ser considerado.

Fica evidente, portanto, a imprescindibilidade da inclusão da espiritualidade na avaliação clínica dos usuários das Unidades de APS, tendo por pressuposto que o preconizado vínculo "médico-paciente" poderá otimizar o acolhimento, a escuta qualificada, a compreensão pelo profissional do significado do adoecer para o paciente, bem como o estabelecimento de um plano terapêutico capaz de considerar as especificidades espirituais e religiosas do paciente, favorecendo dessa forma, o prognóstico e o enfrentamento das adversidades, somáticas ou não, decorrentes do adoecimento.

Esse artigo, por meio de uma revisão de literatura, aborda a necessária inclusão da espiritualidade no cuidado em saúde prestado por profissionais que atuam em Unidades de APS.

\section{Metodologia}

Trata-se de uma revisão do que foipublicado na literatura, na base de dados Scielo e em bibliotecas virtuais no período de 2004 a 2019, utilizando-se os descritores espiritualidade; religião e medicina; relação médico-paciente.

\section{Resultados}

Nas últimas décadas vem-se observando a necessidade de mudança no paradigma do conceito de cuidado em saúde, haja vista que o modelo biomédicocartesiano já não é capaz de explicar a multicausalidade do processo saúde-doença e demandas dos indivíduos que precisam de atenção integral em saúde.

Na prática clínica, na maioria das vezes, não é possível fragmentar o paciente em partes, separando-o em porções social, biológica, psíquica e/ou espiritual. ${ }^{2}$ Tendo em vista que todas estão interligadas e podem ser igualmente responsáveis pela presença decomorbidades, adesão ao plano terapêutico, assim como pelo sucesso ou fracasso no tratamento. Isso se torna evidente no cuidado prestado por profissionais que atuam na APS, que tem entre seus pressupostos, a imprescindibilidade da visão integral do paciente pelo médico, a quem é recomendado a utilização do Método Clínico Centrado na Pessoa (MCCP), integrando e articulando assim, os diversos aspectos da natureza humana. Ressaltam os autores que muitos pacientes, declaradamente religiosos, têm em suas crenças um ponto forte que os ajudam a lidarem com muitos aspectos da vida, incluindo o processo saúde-doença. Justifica-se, portanto, a inclusão da espiritualidade na avaliação clínica dos pacientes pelos profissionais de saúde, o que pode contribuir para uma melhor compreensão de aspectos diagnósticos favorecendo também o prognóstico.

As pesquisas sobre religião, espiritualidade e saúde estão progredindo avassaladoramente, mesmo que significativa parcela de médicos ainda não esteja treinada para a abordagem dessas questões no seu processo de trabalho, evidenciando a necessidade de socialização de estudos sobre a interrelação entre espiritualidade e o cuidado em saúde, de modo que seus resultados possam nortear a prática profissional contribuindo para a resolutividade desse cuidado. ${ }^{8}$

Estudos revelam que as crenças religiosas influenciam o tipo de assistência médica que os pacientes desejam receber e o modo como lidam com a doença. Podem ainda influenciar decisões médicas e a adesão do paciente ao tratamento. Claro está que há muitas razoes pelas quais os profissionais de saúde devem discutir questões religiosas e espirituais com os pacientes. A necessidade de treinamento para incorporar a espiritualidade nos cuidados ao paciente vem sendo reconhecida na educação médica. ${ }^{8}$

Entre as razões que justificam a abordagem da espiritualidade do paciente pelo médico, destacam-se o fato de que muitos pacientes são religiosos e suas crenças ajudam-lhes a lidar com aspectos da vida, assim como crenças pessoais dos médicos influenciam em suas decisões - tanto por parte do paciente como por parte dos médicos. No primeiro caso exemplifica-se o caso de pacientes que são testemunhos de Jeová e não permitem o uso de hemoderivados no tratamento, enquanto a segunda situação pode ser ilustrada pelo caso de médicos que se recusam a prescrever métodos contraceptivos devido a seus princípios religiosos. Pesquisas revelam que, na percepção de número significativo de pacientes, aspectos de sua religião e espiritualidade deveriam ser abordados pelos médicos. Há ainda registro de que pacientes sentiriam mais empatia e confiança pelo médico que incorporou à consulta, a abordagem desses temas, proporcionando o resgate da relação médico e paciente, com uma visão holística e mais humanizada. ${ }^{2}$

Investigações científicas relacionadas à saúde mental indicam menor prevalência de depressão, remissão mais rápida da depressão após tratamento, menor prevalência de ansiedade e menor taxa de suicídio em pacientes que tiveram problematizadas crenças religiosas e espiritualidade pelos profissionais de saúde. Da mesma forma, estudos demonstram uma relação da espiritualidade com melhor qualidade devida e maior bem estar geral. Os pacientes religiosos tiveram menores níveis de hipertensão diastólica, e menores índice de mortalidade por causas cardiovasculares. ${ }^{2}$

No âmbito do cuidado prestado na APS, em especial nas unidades ESF-lócus preferencial do primeiro contato com o serviço de saúde na Rede de Atenção à Saúde - os profissionais de saúde têm contato com pessoas portadoras dos mais diversos problemas, cuja etiologia tem natureza diversa. A doença crônica, o envelhecimento, a solidão, e a possibilidade de finitude da vida, são exemplos de situações vivenciadas pelo ser 
humano que o levam a buscar um encontro consigo e com sua espiritualidade a fim de encontrar forças para superar adoença, solidão e um possível temor da morte, libertando-se numa atitude de transcendência, em uma tentativa de romper limites, de superar e de projetar-se sempre além. A espiritualidade pode ser considerada um apoio na vida, pois através do desenvolvimento da espiritualidade encontra-se apoio para o enfrentamento cotidiano da solidão e da tristeza, gerando amadurecimento por uma vida interior, aceitação das perdas de entes queridos, saída dos filhos de casa, seu envelhecimento da doença e até mesmo de sua finitude. Assim o profissional de saúde precisa proporcionar um cuidado ao ser humano numa perspectiva holística, valorizando o apoio espiritual, visando a que este possa vivenciar momentos com serenidade. Espiritualidade contribuiu para a aceitação dos próprios limites e para a solidariedade com quem necessita.

Relatos interessantes como o de uma Agente Comunitária de Saúde, profissional da equipe que atua na ESF, sobre o fato da existência depessoas que procuram a unidade de saúde, não em busca de um remédio, mas em busca de um conselho, justifica a necessidade de o profissional estar capacitado para prestar outro tipo de cuidado além do procedimento médico intervencionista. É importante, portanto, que os profissionais estejam bem consigo mesmos, para assim poderem ajudar o outro, estimulando um resgate da espiritualidade pelo paciente através do diálogo,

$\mathrm{F}-\mathrm{Fé} /$ crença

- Você se considera religioso ou espiritualizado?

- Você tem crenças espirituais ou religiosas que te ajudam a lidar com problemas?

- Se náo: o que te dá significado na vida?

I - Importåncia ou influência

- Que importància você dá para a fé ou crenças religiosas em sua vida?

- A fé ou crenças já influenciaram você a lidar com estresse ou problemas de saúde?

- Você tem alguma crença específica que pode afetar decisóes médicas ou o seu tratamento?

C - Comunidade

- Você faz parte de alguma comunidade religiosa ou espiritual?

- Ela te dá suporte, como?

- Existe algum grupo de pessoas que você "realmente" ama ou que seja importante para você?

- Comunidades como igrejas, templos, centros, grupos de apoio sáo fontes de suporte importante?

A - Açáo no tratamento

- Como você gostaria que o seu médico ou profissional da área da saúde considerasse a questáo religiosidade / espiritualidade no seu tratamento?

- Indique, remeta a algum lider espiritual / religioso.

Figura 1. Questionário FICA otimizando dessa forma, o desabafo dos sofrimentos e angústias, contribuindo para a resolutividade do cuidado. A desconsideração da dimensão espiritual humana ocasiona prejuízos ao desenvolvimento do capital humano em serviços de saúde. Entre as barreiras a serem transpostas para a abordagem da espiritualidade da prática clínica pelo profissional de saúde, destacam-se a falta de conhecimento sobre o assunto, a inexistência de treinamento, a alegação de falta de tempo pelo profissional, o desconforto com o tema, o medo de impor pontos de vista religiosos ao paciente, a preocupação de atuar em área "não médica",o pensamento de que o conhecimento da religião não é relevante ao tratamento médico, a opinião de que isso não faz parte do papel do médico e, finalmente, a falta de interesse no tema. ${ }^{9,10}$

Não existe uma só forma de abordar a espiritualidade, assim como não existe uma forma correta. Muitas vezes, essa abordagem faz-se de forma natural e tranquila, o que depende das próprias heranças culturais do médico. Entretanto, pesquisadores têm criado formas de facilitar a abordagem da espiritualidade para os médicos que ainda possuem dificuldades com o tema. Existem instrumentos que servem como norteadores para a obtenção da história espiritual.

Os principais instrumentos utilizados, questionários FICA e o HOPE, estão ilustrados nas figuras 1 e 2 , respectivamente. ${ }^{2}$

Os instrumentos "FICA" e "HOPE", que destinam-se à obtenção da história espiritual, estão

H - Fontes de Esperança (Hope), significância, conforto, força, paz, amor e relacionamento social.

- Quais sáo as suas fontes de esperança, força, conforto e paz?

- Ao que você se apega em tempos difíceis?

- O que o sustenta e o faz seguir adiante?

$\mathrm{O}$ - Religiáo organizada

- Você faz parte de uma comunidade religiosa ou espiritual? Ela o ajuda? Como?

- Em que aspectos a religiáo o ajuda e em quais náo o ajuda muito?

P - Espiritualidade pessoal e prática

- Você tem alguma crença espiritual que é independente da sua religiáo organizada?

- Quais aspectos de sua espiritualidade ou prática espiritual você acha que sáo mais úteis à sua personalidade?

E - Efeitos no tratamento médico e assuntos terminais

- Ficar doente afetou sua habilidade de fazer coisas que o ajudam espiritualmente?

- Como médico, há algo que eu possa fazer para ajudar você a acessar os recursos que geralmente $o$ apóiam?

- Há alguma prática ou restriçáo que eu deveria saber sobre seu tratamento médico?

Figura 2. Questionário HOPE 
validados internacionalmente e podem ser incorporados ao processo de trabalho na APS. Mas é preciso ter em mente que um instrumento de avaliação espiritual deve ser de fácil aplicação, flexível, adaptável, curta duração e ser aplicado de modo dialogado com a pessoa. ${ }^{2}$

Assim, há consenso entre vários estudos sobre a importância da abordagem da religiosidade - espiritualidade na anamnese clínica como forma de consolidar a relação médico-paciente e para a construção de uma visão integral das pessoas. O campo de saúde e espiritualidade tem crescido de forma notável nas últimas décadas, impulsionado pelas publicações de alta qualidade e de suas implicações na prática clínica.

Fica evidente que as práticas espirituais têm mostrado resultados passíveis de validação pelas instituições de saúde. Entretanto, existe ainda a necessidade de implementação de um modelo sistematizado para a realização de tratamentos baseados em práticas espirituais e religiosas. ${ }^{12}$

\section{Considerações Finais}

Considerando que a dimensão espiritual tem uma natureza sistêmica, conclui se como fundamental sua incorporação no processo de trabalho dos profissionais que atuam na APS. Há necessidade de uma mudança de paradigma no atendimento a saúde onde o distanciamento de uma visão eminentemente biomédica cartesiana tecnicista ampliará o contexto para a incorporação de visão espiritual, que considere a interface entre espiritualidade e saúde.

\section{Referências}

1. Batista SSP. A espiritualidade na prática do cuidado usuário do programa saúde da família, com ênfase na educação popular em saúde. Revista APSjan/ jun 2007; 10(1):74-80.

2. Lucchetti G, GraneroAL, Bassi RM, Latorraca R, Nacif SAP. Espiritualidade na prática clínica:o que o clínico deve saber? Rev.Bras. ClinMed 2010;8(2): 154-48. Disponível em <http://files.bvs.br/upload/S/1679-1010/2010/v8n2/ a012.pdf $>$ Acesso em 31 de outubro de 2019.

3. PanziniGR. Escala de coping religioso-espiritual (Escala CRE): tradução, adaptação e validação da escala RCOPE, abordando relações com saúde e qualidade de vida. Dissertação.Mestrado em psicologia UFRGS. Outubro de 2004. Disponível em < http://www.amebrasil.org.br/html/raquel.pdf $>$ Acesso em 3 de Novembro de 2019

4. Balboni TA, Vanderwerker LC, Block SD, Paulk ME, Lathan CS, Peteet JR et al.Religiousness and spiritual support among advanced cancer patients and associations with end-of-life treatment preferences and quality of life. J ClinOncol. 2007;25(5):555-60.Disponível em <https:/www.ncbi.nlm.nih. gov/pubmed/17290065> Acesso em 3 de Novembro de 2019.

5. Travado L, Grassi L, Gil F, Martins C, Ventura C, Bairradas J. Do spirituality and faith make a difference? Report from the Southern European Psycho-Oncology Study Group. PalliativeSupportiveCare. 2010;8(4):405413.

6. Mesquita AC, Chaves ÉCL, Avelino CCV, Nogueira DA, Panzini RG, Carvalho EC. A utilização do enfrentamento religioso/espiritual por pacientes com câncer em tratamento quimioterápico. Rev. Latino-Am. Enfermagem. 2013; 21(2):539-545
7. Brasil. Constituição da República Federativa Brasileira. 1988. Disponível em <http://www.planalto.gov.br/ccivil_03/constituicao/constituicao.htm> Acesso em 3 de Novembro de 2019.

8. Koenig GH. Medicina, religião e saúde. O encontro da ciência e da espiritualidade. Porto Alegre: LPM, 2012.

9. Barros Júnior J, Barbosa RS, Luna Neto RT, Tavares NBF, Freitas KM, Cruz CA, Moura UHS. Saúde e Espiritualidade: as narrativas profissionais na estratégia da saúde da família. Revista de Psicologia 2015; 9(26). Disponível em <https://idonline.emnuvens.com.br/id/article/view/329/457> Acesso em 31 de outubro de 2019.

10. Koenig G.H. Espiritualidade no cuidado com o paciente. Porque, como, quando e o que. São Paulo: Associação Medico Espírita de São Paulo, 2018 .

11. Gusso G, Lopes JMC, Dias LC Tratado de medicina de família e comunidade. Princípios, formação e prática. Porto Alegre: Editora Artmed, 2019.

12. Barbosa DJ, Tosoli AMG, Soares GO, Paes LS. Religiosidade e espiritualidade como ferramenta de apoio para o tratamento de usuários de drogas psicoativas. Revista Pró-univerSUS. 2018 Jul./Dez.; 09 (2): 17-23. 